\title{
Estimating Functional Brain Network with Low-Rank Structure via Matrix Factorization for MCI/ASD Identification
}

\author{
Yue Du, Limei Zhang* \\ School of Mathematics Science, Liaocheng University, Liaocheng, China \\ Email: *zhanglimei@lcu.edu.cn
}

How to cite this paper: Du, Y. and Zhang, L.M. (2021) Estimating Functional Brain Network with Low-Rank Structure via Matrix Factorization for MCI/ASD Identification. Journal of Applied Mathematics and Physics, 9, 1946-1963.

https://doi.org/10.4236/jamp.2021.98127

Received: July 15, 2021

Accepted: August 16, 2021

Published: August 19, 2021

Copyright $\odot 2021$ by author(s) and Scientific Research Publishing Inc. This work is licensed under the Creative Commons Attribution International License (CC BY 4.0).

http://creativecommons.org/licenses/by/4.0/ (c) (i) Open Access

\begin{abstract}
Functional brain networks (FBNs) provide a potential way for understanding the brain organizational patterns and diagnosing neurological diseases. Due to its importance, many FBN construction methods have been proposed currently, including the low-order Pearson's correlation (PC) and sparse representation (SR), as well as the high-order functional connection (HoFC). However, most existing methods usually ignore the information of topological structures of FBN, such as low-rank structure which can reduce the noise and improve modularity to enhance the stability of networks. In this paper, we propose a novel method for improving the estimated FBNs utilizing matrix factorization (MF). More specifically, we firstly construct FBNs based on three traditional methods, including PC, SR, and HoFC. Then, we reduce the rank of these FBNs via MF model for estimating FBN with low-rank structure. Finally, to evaluate the effectiveness of the proposed method, experiments have been conducted to identify the subjects with mild cognitive impairment (MCI) and autism spectrum disorder (ASD) from norm controls (NCs) using the estimated FBNs. The results on Alzheimer's Disease Neuroimaging Initiative (ADNI) dataset and Autism Brain Imaging Data Exchange (ABIDE) dataset demonstrate that the classification performances achieved by our proposed method are better than the selected baseline methods.
\end{abstract}

\section{Keywords}

Functional Brain Network, Matrix Factorization, Pearson's Correlation, Sparse Representation, High-Order Functional Connection, Mild Cognitive Impairment, Autism Spectrum Disorder 


\section{Introduction}

Functional brain network (FBN), calculated by resting-state functional magnetic resonance imaging (rs-fMRI), can provide informative pattern about the organization of the brain [1], and mine sensitive biomarkers for neurological or psychological diseases such as Autism spectrum disorder (ASD) [2] [3], Parkinson's disease [4], Alzheimer's disease (AD) [5] [6] and its early stage, i.e., mild cognitive impairment (MCI) [7] [8] [9]. All of the disease identification tasks rely on the quality of the FBNs, and therefore it is indispensable to estimate more reasonable FBNs.

To date, researchers have developed many FBN estimation methods to improve the accuracy of disease prediction [10] [11]. Specifically, Pearson's correlation (PC) is the most popular and simplest method for FBN estimation that measures the full correlation between different brain regions [12]. Despite its simplicity, high computational efficiency and statistical robustness, the full connection FBN tends to include confounding effects from other brain regions. By contrast, the complex interactions between multiple regions-of-interest (ROIs) can be explained by partial correction or its $l_{1}$-regularized version, namely sparse representation (SR).

Compared with the low-order FBN estimation methods, such as PC and SR, some high-order statistics may also offer additional and useful information for FBN analysis [13] [14] [15]. For example, Zhang et al. [16] recently proposed a high-order FBN (HoFBN) estimation method that can effectively find identify biomarkers between different groups of subjects. To put it easy to understand, we consider the relationship network as an analogy, as shown in Figure 1, where the nodes denote persons, the edges are the relationship between the persons, and the weight on each edge is the strength of relationship. In this example, the relationship provides the low-order connection information of the network. However, there may exist some relationships among the friendship network of one person which may provide some high-order connection information for a network system. However, the three traditional methods do not take the

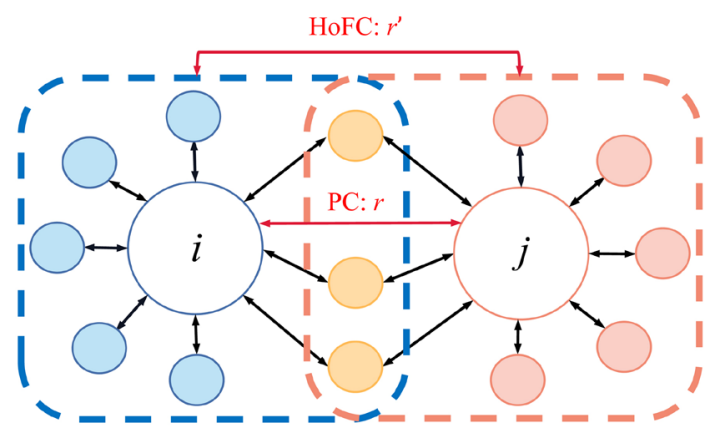

Figure 1. The blue and red nodes are the friends of the $i$-th node and $j$-th node respectively, and the yellow nodes represent the common friends of nodes $i$ and $j$. The blue and red boxes are the friendship networks of $i$ and $j . r$ is the low-order relationship between $i$ and $j$, and the high-order relationship between $i$ and $j$ is expressed by $r^{\prime}$ which is measured via the friendship network of $i$ and $j$. 
topological structure of the FBN into account. It has been reported that FBN usually has modularity structure [17] [18] [19], meaning that the network exists some node modules, and the nodes are connected densely in each module, while the connections between these modules are sparse. In consequence, the nodes within a same module tend to have a high probability connect to each other, which may lead to some dependent rows/columns and further resulting in a low-rank edge weight matrix. However, most methods for estimating FBN, such as PC, SR, and HoFC, usually produce full-rank networks, indicating that there are some noises in these networks.

For improving the quality of the FBN estimated by conventional methods, we propose a novel FBN construction method that uses matrix factorization (MF) to characterize the low-rank structure, while preserving the information of traditional FBN as much as possible. Specifically, first, we estimate FBNs for all the subjects using PC, SR and HoFC, and then we perform the MF of the estimeted FBNs to obtain a new connections network with $r a n k=k$. Finally, to evaluate the effectiveness of the proposed method, we conduct the experiments based on two public database (Alzheimer's Disease Neuroimaging Initiative (ADNI) dataset and Autism Brain Imaging Data Exchange (ABIDE) dataset). The experimental results show that our method can effectively improve the identification accuracy compared with the baseline methods.

The rest of this paper is organized as follows. In Section 2, we review three conventional FBN estimation methods, i.e. PC, SR, and HoFC. In Section 3, we first introduce the datasets used in this study and pre-processing pipeline. Then we present our proposed method to estimate FBNs and solving process. In Section 4, we exhibit our experimental setting and the results for classification. In Section 5 , we investigate the influence of modeling parameters, number of rank on the final classification performance. Beside, we also discuss the modularity score of FBNs and discriminative functional connections for MCI identify and several limitations of this work as well as the possible research in the future directly. Finally, we briefly summarize this paper in Section 6.

\section{Related Work}

By reason of the significant role in exploring the inherent organization and neurodegenerative diseases of the brain, researchers have proposed many FBN estimation methods in the past decades. In this section, we review several methods, i.e. PC [20], SR [21], and HoFC [16], which are closely related to our study briefly.

\subsection{Pearson's Correlation}

It is well-known that PC is the simplest and most popular method for FBN estimation. Its mathematical expression is given as follows:

$$
w_{i j}=\frac{\left(x_{i}-\bar{x}_{i}\right)^{\mathrm{T}}\left(x_{j}-\bar{x}_{j}\right)}{\sqrt{\left(x_{i}-\bar{x}_{i}\right)^{\mathrm{T}}\left(x_{i}-\bar{x}_{i}\right)} \sqrt{\left(x_{j}-\bar{x}_{j}\right)^{\mathrm{T}}\left(x_{j}-\bar{x}_{j}\right)}},
$$


where $x_{i} \in R^{m}$ is the blood oxygen level-dependent (BOLD) signal associated with the $i^{\text {th }}$ ROI, $m$ is the total number of temporal image volumes, and $\bar{x}_{i} \in R^{m}$ is the mean of the elements in $x_{i}$. Without loss of generality, we redefine $x_{i}$ by centralization and normalization like $x_{i}=\left(x_{i}-\bar{x}_{i}\right) / \sqrt{\left(x_{i}-\bar{x}_{i}\right)^{\mathrm{T}}\left(x_{i}-\bar{x}_{i}\right)}$. Then, Equation (1) can be simplified as $w_{i j}=x_{i}^{\mathrm{T}} x_{j}$, which can be easily proved to be the optimal solution of the following optimization problem [22]:

$$
\min _{w_{i j}} \sum_{i, j}^{n}\left\|x_{i}-w_{i j} x_{j}\right\|^{2},
$$

or its matrix form:

$$
\min _{W}\left\|W-X^{\mathrm{T}} X\right\|_{F}^{2},
$$

where $W$ is the adjacency matrix to be estimate by PC, $X=\left[x_{1}, x_{2}, \cdots, x_{n}\right] \in R^{m \times n}$ denotes the data matrix which is the set of BOLD signals, and $n$ is the number of ROIs.

\subsection{Sparse Representation}

SR is another commonly utilized method to estimate FBNs which regressing out the confounding effect from other ROIs, and the mathematical model of SR is expressed as follows:

$$
\begin{array}{ll}
\min _{w_{i j}} & \sum_{i=1}^{n}\left(\left\|x_{i}-\sum_{j \neq i} w_{i j} x_{j}\right\|^{2}+\lambda \sum_{j \neq i}\left|w_{i j}\right|_{1}\right) \\
\text { s.t. } \quad w_{i i}=0, \forall i=1, \cdots, n,
\end{array}
$$

where $\lambda$ is the regularized parameter for controlling the balance of two terms in Equation (4). Mathematically, Equation (4) can be further rewritten by the following matrix form:

$$
\begin{array}{ll}
\min _{W} & \|X-X W\|_{F}^{2}+\lambda\|W\|_{1} \\
\text { s.t. } & w_{i i}=0, \forall i=1, \cdots, n,
\end{array}
$$

where $\|X-X W\|_{F}^{2}$ is a data fitting term which implies to invert the covariance matrix and capture the information of partial correction, $\|W\|_{1}$ denotes $l_{1}$ -regularized term which encoding the sparsity prior of FBN. Note that, the constraint $w_{i i}=0$ is to avoid the trivial solution.

\subsection{High-Order Functional Connectivity}

As mentioned in Section 1, many HoFBN estimation methods has been proposed in recently years [14] [15] [16]. In this work, we only review the high-order functional connectivity (HOFC) due to it is simplest and direct. The HoFC method includes two steps (as shown in Figure 2): First, computing the low-order correlation coefficient $r_{i j}$ based on the BOLD signal corresponded by $i$-th and $j$-th ROI utilize PC. Then, HOFC between brain regions $i$ and $j, r_{i j}^{\prime}$, can be calculated by the pearson's correlation coefficient between $i$-th row (or column) and $j$-th row (or column) in the low-order network. 


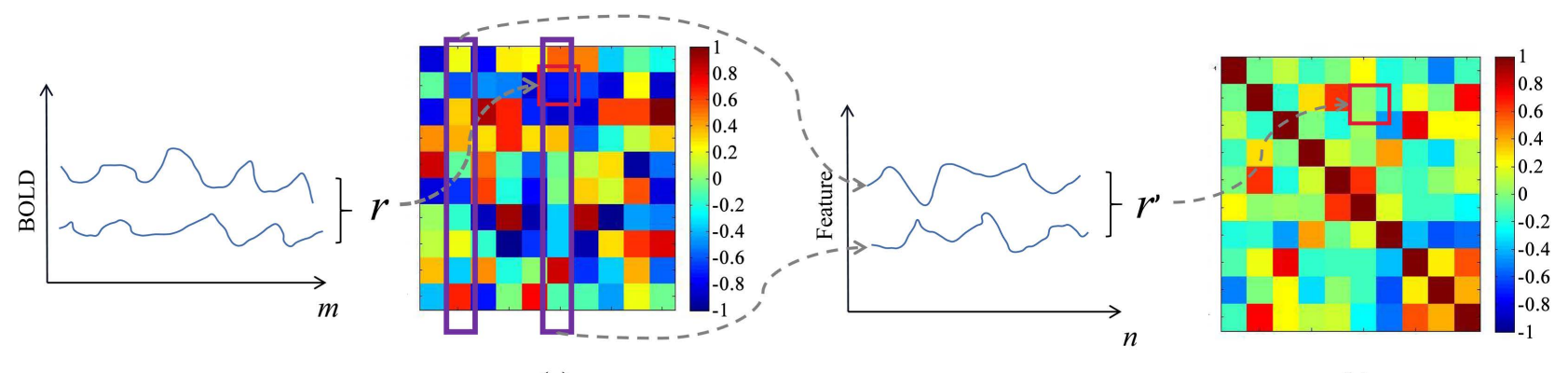

(a)

(b)

Figure 2. Pipeline of the estimation of HoFBN use HoFC. (a) PC; (b) Ho-FC.

The model is as follows:

$$
r_{i j}^{\prime}=\frac{\left(L_{i}-\bar{L}_{i}\right)^{\mathrm{T}}\left(L_{j}-\bar{L}_{j}\right)}{\sqrt{\left(L_{i}-\bar{L}_{i}\right)^{\mathrm{T}}\left(L_{i}-\bar{L}_{i}\right)} \sqrt{\left(L_{j}-\bar{L}_{j}\right)^{\mathrm{T}}}\left(L_{j}-\bar{L}_{j}\right)},
$$

where $L_{i}$ is the $i$-th row (or column) in low-order network.

\section{Materials and Methodology}

In this section, we design a framework for brain disease classification, as shown in Figure 3. Including the data preparation, the proposed method, and the MCI/ASD classification pipeline.

\subsection{Data Preparation}

To verify the availability of the proposed method, we use two benchmark databases, i.e., ADNI dataset and ABIDE dataset in the experiment.

For ADNI dataset, 137 subjects (including 68 MCIs and 69 NCs.) were extracted and preprocessed in [23]. Table 1 shows the demographic information of these 137 subjects. For each subject, the scanning time was $7 \mathrm{~min}$, corresponding to 140 volumes. The head motion, brain size, shape, orientation, gyral anatomy, the differences in image acquisition time between slices, long-term physiological shifts, and instrumental instability have large influence on the fMRI reliability. In order to reduce these influences, a preprocessing pipeline is used in this paper to improve fMRI data quality prior to FBN estimation. At the beginning, the first 3 volumes were removed for signal stabilization, and then processed the remaining 137 volumes via a standard pipeline (the Data Processing Assistant for Resting-State fMRI (DPARSF) toolbox.) [24]. Specifically, the subjects which with more than 2.5 min of FD (alculated based on head motion parameters), larger than $0.5 \mathrm{~mm}$ were removed. Then, we reduce the influence of the ventricle and white matter signals used nuisance regression as well as the high-order effect of head motion based on Friston 24-parameters model. After that, we performe the fMRIs onto the Montreal Neurological Institute (MNI) space, followed by spatially smoothing with the full-width-half-maximum of $4 \mathrm{~mm}$. Finally, every brain were divided into 116 ROIs based on the Automated Anatomical Labeling 


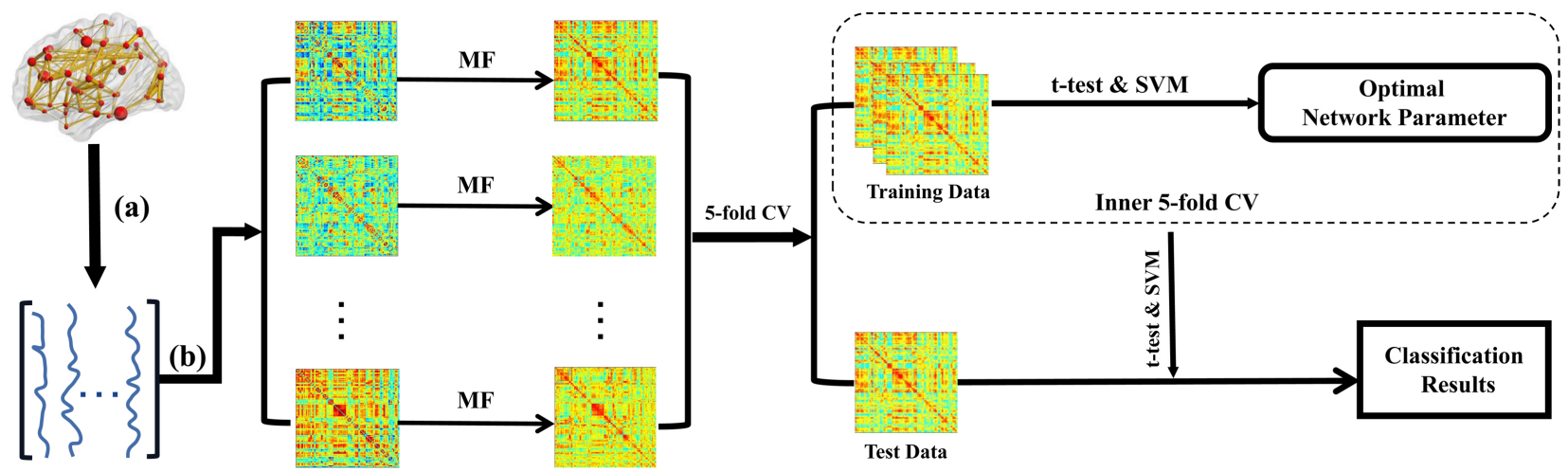

Figure 3. The main pipeline of MCI/ASD identification used in this study, which contains three major steps: (a) data preparation, (b) FCN construction, and (c) classification with feature selection.

Table 1. Demographic and clinical information of subjects in the ADNI and ABIDE datasets. Values are reported as mean \pm standardard deviation. M/F: Male/Female; MMSE: Mini-Mental Examination; GCDR: Global Clinical Dementia Rating; FIQ: Full-Scale Intelligence Quotient; VIQ: Verbal Intelligence Quotient; PIQ: Performance Intelligence Quotient.

\begin{tabular}{ccccccccc}
\hline Datasets & Class & Gender (M/F) & Age (Years) & MMSE & GCDR & FIQ & VIQ & PIQ \\
\hline \multirow{2}{*}{ ADNI } & MCI & $39 / 29$ & $76.50 \pm 13.50$ & $26.77 \pm 1.23$ & $0.48 \pm 0.02$ & - & - & - \\
& NC & $17 / 52$ & $71.50 \pm 14.50$ & $28.85 \pm 1.15$ & 0 & - & - & - \\
& ASD & $68 / 11$ & $18.58 \pm 11.45$ & - & - & $107.92 \pm 3.15$ & $105.81 \pm 1.23$ & $108.81 \pm 2.10$ \\
\multirow{2}{*}{ ABIDE } & NC & $79 / 26$ & $19.13 \pm 11.85$ & - & - & $113.15 \pm 2.45$ & $113.13 \pm 1.15$ & $115.07 \pm 2.08$ \\
& & & & & & & &
\end{tabular}

(AAL) template [25], and we extracted the mean time series (with band-pass filtered $0.015-0.150 \mathrm{~Hz}$ ) of each ROIs as the input data of the proposed method.

For the ABIDE dataset, we used 184 subjects (including 79 ASDs and 105 NCs) that are from the largest site New York University (NYU) in this work. The demographic information of these subjects are also shown in Table 1. Specifically, the fMRI data were acquired based on a standard echo-planar imaging sequence on a clinical routine 3.0 Tesla Allegra scanner with the following imaging parameters: TR/TE is $2000 / 15 \mathrm{~ms}$ with 180 volumes, the number of slices is 33 , and the slice thickness is $4.0 \mathrm{~mm}$. The involved fMRI data are provided by the Preprocessed Connectome Project initiative, and further preprocessed by DPARSF. More specifically, the preprocessing pipeline includes four mainly steps: 1) volume slices and head motion correction, 2) nuisance signals regression, 3) registration to MNI space, and 4) temporal filtering $(0.01-0.10 \mathrm{~Hz})$. Afterwards, the brain of each subject is partitioned into 116 ROIs used AAL atlas, and extracted mean time series from all these ROIs for constituting the data matrix $X \in R^{175 \times 116}$.

\subsection{Proposed Method for FBN Estimation}

To further improve the identifiability of the FBNs, we propose a low-rank FBN estimation method by MF to reduce the rank of the network which is estimated by the conventional methods. There are two motivation for our study: 1) Low-rank structure is beneficial to reduce the noise which disturb the diagnosis of the 
network, thereby obtaining a more "clear" FBN to improve the accuracy of MCI/ASD identification. 2) As reported in the study Qiao et al. [26], low-rank structure can enhance the modularity of network which is extremely important for promoting stability of our brain. The rank of the network can be reduced by MF effectively which has a elegant probability interpretation and optimal solution, and the model as follow:

$$
\min _{U, V \in R^{n \times k}}\left\|W-U V^{\mathrm{T}}\right\|_{F}^{2}+\mathbb{L}(U, V),
$$

where $W$ is the network estimated by traditionary methods, $k$ is the rank of the FBNs estimated by the proposed method. $\left\|W-U V^{\mathrm{T}}\right\|_{F}^{2}$ is the data fitting term for maintaining as much information of $W . \mathbb{L}(U, V)$ is the matrix-regularized term which is usually necessary in prediction for bias-variance trade-off [27]. Further, make the model concrete:

$$
\min _{U, V \in R^{n \times k}}\left\|W-U V^{\mathrm{T}}\right\|_{F}^{2}+\alpha\|U\|_{F}^{2}+\beta\|V\|_{F}^{2},
$$

note that, we use Frobenius Norm since it has a Guassian noise interpretation, and Equation (8) can be easily transformed into the following matrix form:

$$
\min _{U, V \in R^{n \times k}} \operatorname{Tr}\left(W W^{\mathrm{T}}+V U^{\mathrm{T}} U V^{\mathrm{T}}-2 W^{\mathrm{T}} U V^{\mathrm{T}}\right)+\alpha \operatorname{Tr}\left(U^{\mathrm{T}} U\right)+\beta \operatorname{Tr}\left(V^{\mathrm{T}} V\right) .
$$

We take the derivative of $U$ and $V$ respectively:

$$
\begin{aligned}
\frac{\partial}{\partial U} & =\frac{\partial \operatorname{Tr}\left(V U^{\mathrm{T}} U V^{\mathrm{T}}-2 W^{\mathrm{T}} U V^{\mathrm{T}}\right)+\alpha \operatorname{Tr}\left(U^{\mathrm{T}} U\right)}{\partial U} \\
& =\frac{\partial \operatorname{Tr}\left(U^{\mathrm{T}} U V^{\mathrm{T}} V-2 U V^{\mathrm{T}} W^{\mathrm{T}}\right)+\alpha \operatorname{Tr}\left(U^{\mathrm{T}} U\right)}{\partial U} \\
& =2\left(U V^{\mathrm{T}} V-W V+\alpha U\right) . \\
\frac{\partial}{\partial V} & =\frac{\partial \operatorname{Tr}\left(V U^{\mathrm{T}} U V^{\mathrm{T}}-2 W^{\mathrm{T}} U V^{\mathrm{T}}\right)+\beta \operatorname{Tr}\left(V^{\mathrm{T}} V\right)}{\partial V} \\
& =\frac{\partial \operatorname{Tr}\left(V^{\mathrm{T}} V U^{\mathrm{T}} U-2 V^{\mathrm{T}} W^{\mathrm{T}} U\right)+\beta \operatorname{Tr}\left(V^{\mathrm{T}} V\right)}{\partial V} \\
& =2\left(V U^{\mathrm{T}} U-W^{\mathrm{T}} U+\beta V\right) .
\end{aligned}
$$

And then, Equation (9) can be efficiently solved by alternatively update $U$ and $V$ in each iteration of gradient descent algorithm based on Equation (10) and Equation (11). $W^{*}=V * U^{\mathrm{T}}$ is the low-rank FBN constructed by the proposed method.

\subsection{FBN-Based Disease Classification}

After constructing the FBNs of all subjects, the subsequently work is to classify MCIs (or ASDs) and NCs based on these FBNs. To be specific, the first task is to select the features for identification procedure. In our experiment, 6670 features can be produced, since the adjacency matrix of the estimated FBN is symmetric. Thus, the feature dimension is very high compared with the sample size. It typi- 
cally causes the curse of dimensionality which generate the classification accuracy with false enhancement [28]. To address this issue, numerous feature selection methods have been proposed such as $t$-test, least absolute shrinkage and selection operator (LASSO) [29], genetic algorithm (GA) [30] and so on. In this work, we only adopt the simple feature selection method, i.e. $t$-test, and fixed $p=0.05$ empirically. In the second step, we utilize the linear Support Vector Machine (SVM) [31] classifier ( $C=1$ ) since the classifier design has an enormous influence on the ultimate accuracy [32]. Specifically, we use the linear kernel function of SVM, i.e., $\kappa(x, z)=x \cdot z$. There are two reasons for using the linear kernel function: 1 ) Since more features (usually more than the number of subjects) are used in our classification task, SVM with linear kernel function can achieve a relatively ideal classification effect. 2) The linear kernel function has fewer parameters and the computational efficiency is higher than the complex kernel function. We choose 5-fold cross validation (5-fold CV) to evaluate the performance of involved methods.

\section{Experiment}

In this section, we introduce the primary experimental procedure, including the competing methods in this work, the experimental setup and the classification results for different methods.

\subsection{Competing Methods}

As mentioned in Section 3, after obtaining the pre-processed fMRI data, we extracted mean signal for each ROI as the input data to estimate FBNs based on different methods, including: 1) the conventional methods, i.e. PC, SR and HoFC. 2) LR-PC, LR-SR and LR-HoFC use our proposed method to reduce the rank of the FBN estimated by PC, SR and HoFC.

\subsection{Experimental Setup}

In our experiments, to verify the effectiveness of the proposed method, we use 5-fold CV to evaluate different methods and repeat 100 times since 5-fold CV usually generate an unstable value. Note that, for a fair comparison, the same linear SVM (with $C=1$ ) is used in all the methods. Since the modeling parameters may significantly affect the structure of the constructed networks and further influence the ultimate classification results, we select optimal parameters through a large-scale grid search. Specifically, we uniformly utilize 11 candidate values $\left[2^{-5}, 2^{-4}, \cdots, 2^{0}, \cdots, 2^{5}\right]$ for the regularization parameters $(\lambda, \alpha$ and $\beta$ ) in SR, LR-PC, LR-SR and LR-HoFC. In particular, the proposed method has a hidden parameter $k$ which is the rank of the estimated low-rank FBNs. We first assign a value to $k$ empirically, and then we will discuss the value of $k$ in the range of $[10,20, \cdots, 110]$ in Section 5 . To be consistent with other methods, we use 11 candidate values $[100 \%, 90 \%, \cdots, 10 \%, 1 \%]$ when building FBN base on PC and LR-HoFC. For example, 100\% means all edges are preserved, and 90\% means $10 \%$ weak edges are removed. 


\subsection{Estimated Functional Brain Networks}

In this section, we visualize the FBNs estimated by six different methods (i.e., PC, SR, HoFC, LR-PC, LR-SR and LR-HoFC) base on a random subject from $\mathrm{ADNI} / \mathrm{ABIDE}$ dataset. The results are shown in Figure 4, and the parameters are $\lambda=2^{5}$ for SR, $\alpha=2^{4}, \beta=2^{3}$ for LR-PC, LR-SR and LR-HoFC. Based on Figure 4, it can be observer that the FBN estimated by LR-PC, LR-SR and LR-HoFC are cleaner than the baseline methods, respectively. This means that the proposed method may improve the quality of FBNs by reducing possible noisy edges. Besides, FBNs estimated by our proposed method have higher modularity structure compared with other results, and then we will detailed discuss in Section 5.

\subsection{Classification Results}

In this section, we report the classification performances of different methods under five evaluation metrics, i.e., accuracy (ACC), specificity (SPE), sensitivity (SEN), $F_{1}$ and AUC (the area under the receiver operating characteristic (ROC) curve), which mathematical definitions are given as follows:

$$
\begin{gathered}
\text { Accuracy }=\frac{\mathrm{TP}+\mathrm{TN}}{\mathrm{TP}+\mathrm{FP}+\mathrm{TN}+\mathrm{FN}} \\
\text { Specificity }=\frac{\mathrm{TN}}{\mathrm{FP}+\mathrm{TN}} \\
\text { Sensitivity }=\frac{\mathrm{TP}}{\mathrm{TP}+\mathrm{FN}} \\
\mathrm{F}_{1}=\frac{2 \mathrm{TP}}{2 \mathrm{TP}+\mathrm{FN}+\mathrm{FP}}
\end{gathered}
$$

where TP, TN, FP and FN represent true positive, true negative, false positive and false negative.

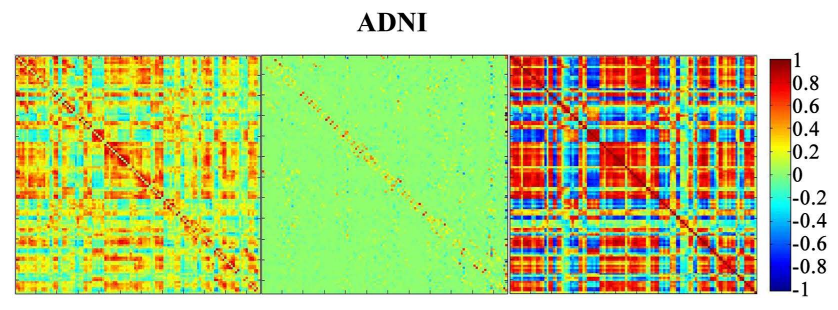

(a)

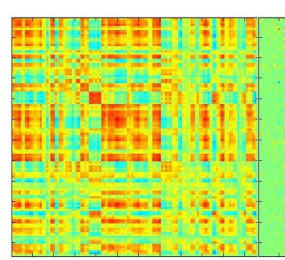

(d) (b)

(c)

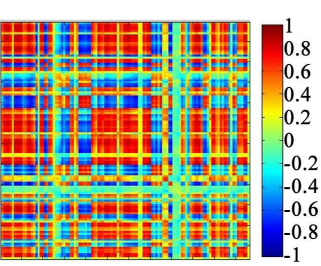

(f)

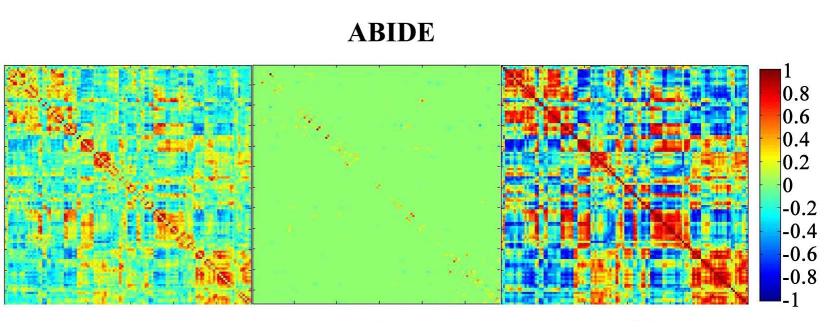

(a)

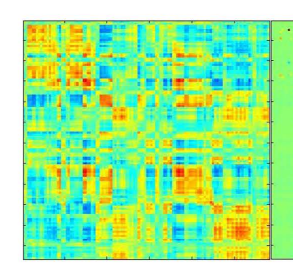

(d) (b)

(c)

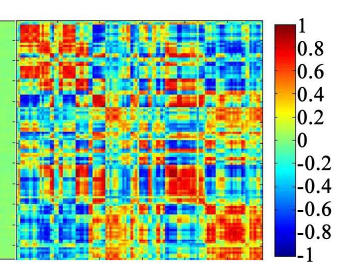

(f)

Figure 4. The adjacency matrices of the same subject estimated by six different methods, i.e., (a) PC, (b) SR, (c) HoFC, (d) LR-PC, (e) LR-SR and (f) LR-HoFC. 
In Table 2, we report the classification results achieved by six different ways. 1) Results of MCI identification: We found that the performances of LR-PC, LR-SR and LR-HoFC are better than PC, SR and HoFC, respectively. Further, the terms marked by "*” denote that the result of the proposed method is significantly better than that of conventional methods under the assumption of $p<0.05$. Specifically, the LR-PC and LR-SR are significantly better than PC and $\mathrm{SR}$ in the terms of all the five evaluation metrics, and LR-HoFC is significantly better than HoFC in the terms of ACC, SEN and $F_{1} .2$ ) Results of ASD identification: It can be seen from Table 2 that the proposed method comprehensively outperforms the competing methods in terms of ACC, SEN, $F_{1}$ and AUC. Only in SPE, LR-SR is slightly lower than SR. In particular, LR-HoFC achieves the significantly better performance than HoFC in the sense of all the five evaluation metrics. LR-PC and LR-SR obtain significantly better results of four indicator respectively. The results in Table 2 illustrate that the low-rank structure introduced by the proposed method not only can remove the "noise", but also can enrich the topological structure of FBNs, and thus enhancing the discrimination of the estimated FBNs. In addition, we report the algorithm running time of the four methods in Table 3. It is worth noting that our method achieve the shortest running time, which shows that the proposed method is simple and can work with high efficiency.

Table 2. Classification performance (mean \pm standard deviation) of 6 different methods in MCI vs. NC and ASD vs. NC classification. The term marked by “*” denotes that the result of proposed methods is significantly better than that of the baseline methods (with $p<0.05$ ). The best results in each pair of Comparisons are shown in bold.

\begin{tabular}{|c|c|c|c|c|c|c|}
\hline Dataset & Method & ACC & SEN & SPE & $\mathrm{F}_{1}$ & $\mathrm{ACU}$ \\
\hline \multirow{6}{*}{$\mathrm{ADNI}$} & PC & $0.7651 \pm 0.0252$ & $0.7652 \pm 0.0355$ & $0.7708 \pm 0.0313$ & $0.7668 \pm 0.0662$ & $0.8289 \pm 0.0285$ \\
\hline & LR-PC & $0.8219 \pm 0.0122^{*}$ & $0.8235 \pm 0.0120^{*}$ & $0.8510 \pm 0.0210^{*}$ & $0.9679 \pm 0.0302^{*}$ & $0.8794 \pm 0.0192^{*}$ \\
\hline & SR & $0.7469 \pm 0.0095$ & $0.7477 \pm 0.0278$ & $0.7643 \pm 0.0252$ & $0.6014 \pm 0.0410$ & $0.8489 \pm 0.0162$ \\
\hline & LR-SR & $0.8301 \pm 0.0192^{*}$ & $0.8444 \pm 0.0252^{*}$ & $0.8167 \pm 0.0303^{*}$ & $0.9097 \pm 0.0466^{*}$ & $0.9096 \pm 0.0125^{*}$ \\
\hline & HoFC & $0.7337 \pm 0.0273$ & $0.7079 \pm 0.0381$ & $0.7606 \pm 0.0240$ & $0.6048 \pm 0.0296$ & $0.7894 \pm 0.0534$ \\
\hline & LR-HoFC & $0.7603 \pm 0.0053^{*}$ & $0.7976 \pm 0.0081^{*}$ & $0.7209 \pm 0.0103$ & $0.8000 \pm 0.0011^{*}$ & $0.8184 \pm 0.0025$ \\
\hline \multirow{6}{*}{ ABIDE } & PC & $0.6560 \pm 0.0131$ & $0.6138 \pm 0.0204$ & $0.6834 \pm 0.0239$ & $0.6130 \pm 0.0398$ & $0.7421 \pm 0.0246$ \\
\hline & LR-PC & $0.7167 \pm 0.0152^{*}$ & $0.6938 \pm 0.0259^{*}$ & $0.7384 \pm 0.0183^{*}$ & $0.6667 \pm 0.0291^{*}$ & $0.7578 \pm 0.0142$ \\
\hline & SR & $0.6216 \pm 0.0114$ & $0.3759 \pm 0.0375$ & $0.8253 \pm 0.0434$ & $0.4336 \pm 0.0225$ & $0.6339 \pm 0.0194$ \\
\hline & LR-SR & $0.7117 \pm 0.0212^{*}$ & $0.5722 \pm 0.0292^{*}$ & $0.8128 \pm 0.0442$ & $0.6471 \pm 0.0105^{*}$ & $0.7596 \pm 0.0157^{*}$ \\
\hline & HoFC & $0.6182 \pm 0.0179$ & $0.5222 \pm 0.0201$ & $0.6947 \pm 0.0209$ & $0.5445 \pm 0.0718$ & $0.6710 \pm 0.0211$ \\
\hline & LR-HoFC & $0.6742 \pm 0.0045^{*}$ & $0.6044 \pm 0.0071^{*}$ & $0.7320 \pm 0.0056^{*}$ & $0.6270 \pm 0.0002^{*}$ & $0.8184 \pm 0.0025^{*}$ \\
\hline
\end{tabular}

Table 3. The algorithm running time of different FBN estimation methods.

\begin{tabular}{ccccc}
\hline Time(s) Method Dataset & PC & SR & HoFC & Ours \\
\hline ADNI & 4.924 & 269.313 & 0.903 & 0.708 \\
ABIDE & 5.632 & 281.804 & 1.192 & 1.024 \\
\hline
\end{tabular}




\section{Discussion}

Specifically, we study the sensitivity of modeling parameters, the influence of the number of rank in MCI classification, and we show the discriminative features visually. We also compared the modularity score obtained by our method with that obtained by traditional methods and list several limitations of our work.

\subsection{Sensitivity to Modeling Parameters}

Proverbially, FBN estimation models usually involve the free parameters which significantly affect the FBN structure. In Figure 5, we exhibit the ACC (using leave-one-out (LOO) cross validation) of six methods with different parametric combinations ( $k=50, k=30$ and $k=30$ for LR-PC, LR-SR and LR-HoFC, respectively). It can be seen that the classification accuracy of PC, SR and HoFC are exceedingly sensitive to the parametric value [33] [34]. In contrast, the results of LR-PC, LR-SR, and LR-HoFC are relatively stable, which means that the proposed method has better robustness. In addition, we can achieve the best ACC in all three sets of comparisons under the optimal parameter.

\subsection{Influence of Number of Rank}

As the complexity and unknown of functional brain network, the optimal value of the parameter $k$ is a practical problem. Thus, we report the five performance metrics achieved by LR-PC, LR-SR and LR-HoFC with different values of $k$ in Figure 6 to investigate the influence of the number of $k$ on the classification results. It can be observed in Figure 6 that LR-PC, LR-SR and LR-HoFC achieve the best performance with $k=50, k=30$ and $k=30$, respectively. Thus, we suspect that FBN achieve the best quality when $30 \leq k \leq 50$. If $k<30$, the

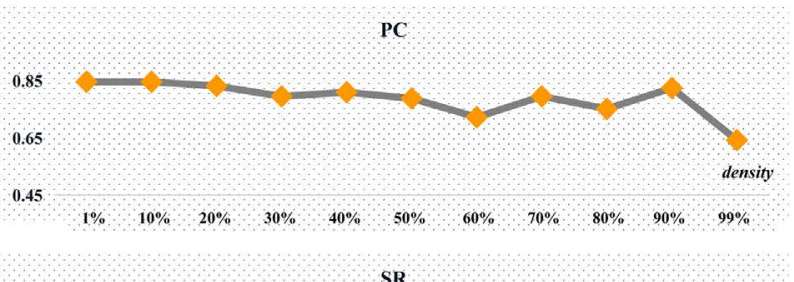

SR

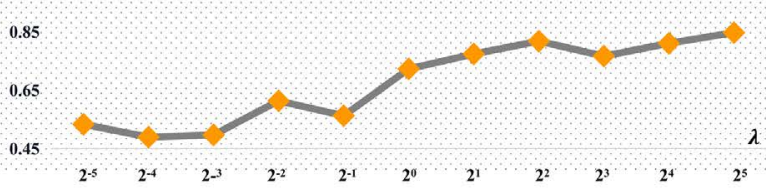

$\mathrm{HoFC}$

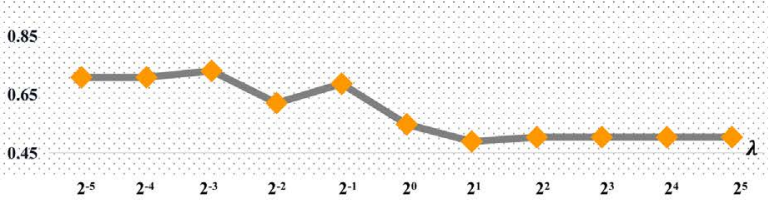

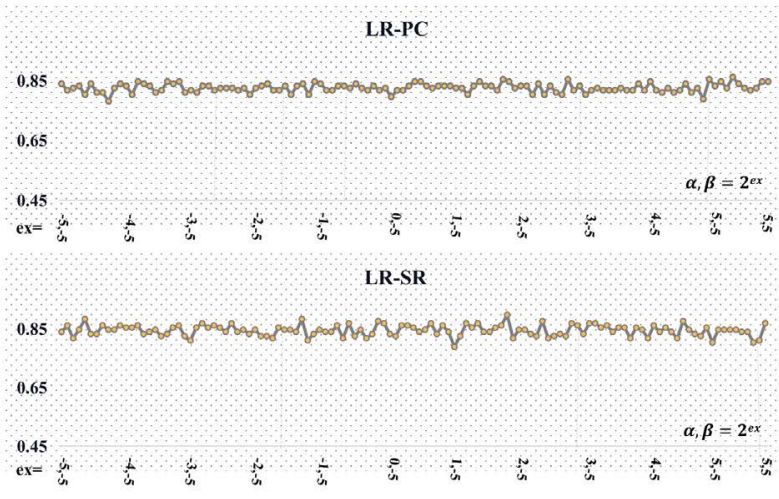

LR-HOFC

0.85

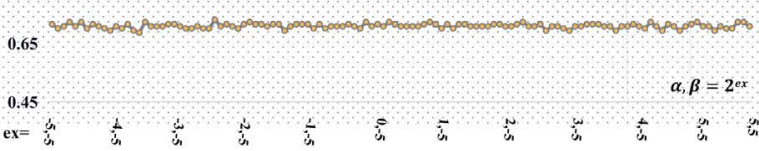

Figure 5. The classification accuracy based on FBNs that estimated by six different methods with 121 or 11 parametric combinations. 


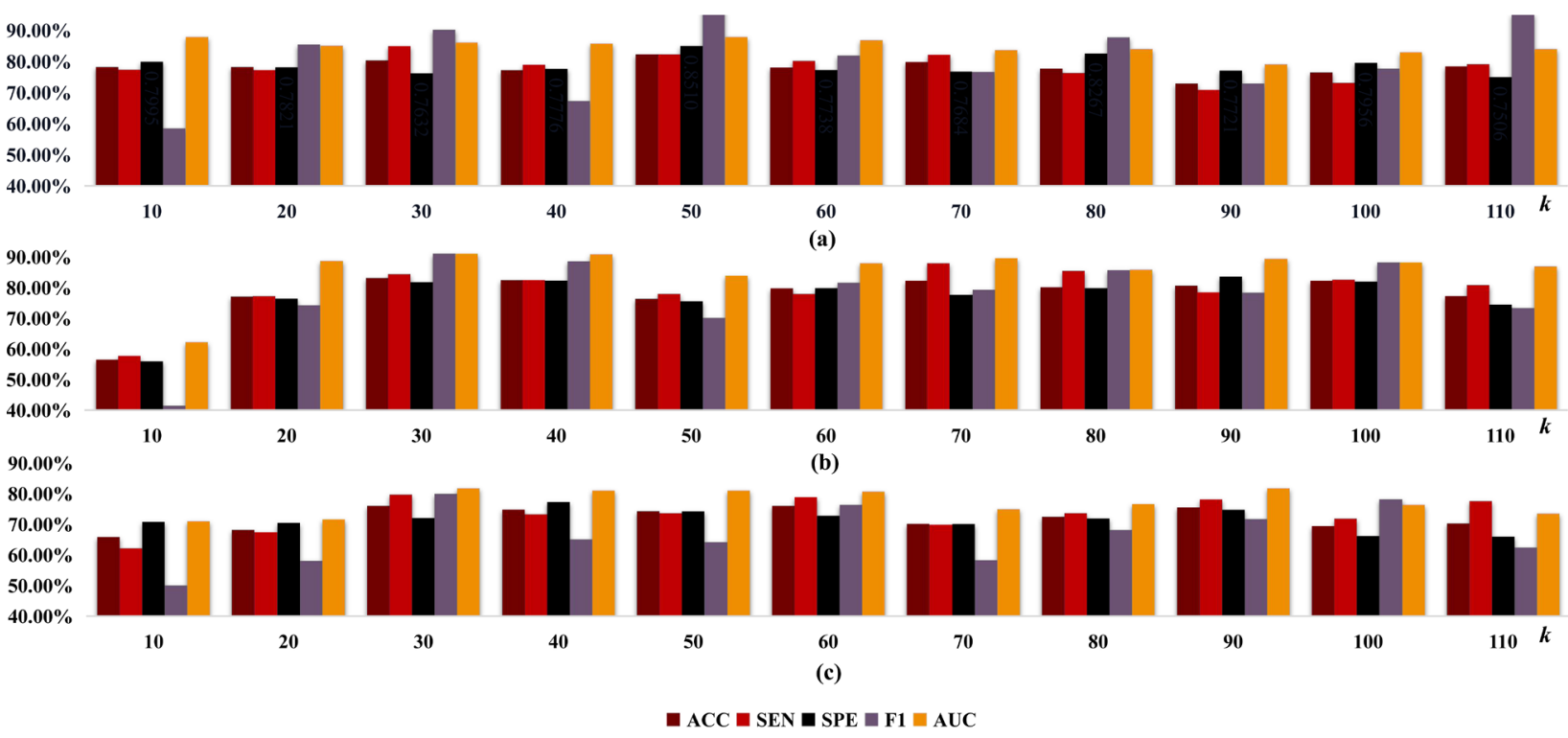

Figure 6. The ACC, SEN, SPE, $\mathrm{F}_{1}$ and AUC values of our methods (i.e. LR-PC, LR-SR and LR-HoFC) with different numbers of the rank of FBNs (i.e., $k$ ) in the task of MCI vs. NC classification. (a) LR-PC; (b) LR-SR; (c) LR-HoFC.

information in the networks may be excessively lost; if $k>50$, there may have a certain degree noise in the network.

\subsection{Discriminative Functional Connections}

For further illustrate the effectiveness of our proposed method, we investigate the most discriminative connections based on the proposed LR-PC, LR-SR and LR-HoFC, respectively. Here, constructing FBNs by LR-PC with $\alpha=2^{5}$, $\beta=2^{-1}$, LR-SR with $\alpha=2^{1}, \beta=2^{5}$, and LR-HoFC with $\alpha=2^{-3}, \beta=2^{1}$. And further the most discriminative features (31, 32 and 41 features for LR-PC, LR-SR and LR-HoFC) are selected with $p=0.01$. As visualized in Figure 7, the discrimination of features is represented by the thickness of the corresponding arc.

In Figure 7, we note that several ROIs that marked by red boxes, including middle temporal gyrus, supramarginal gyrus, amygdalae, hippocampus, precuneus and superior-medial frontal gyrus, are selected in our proposed methods. Especially, hippocampus is identified in all three methods, indicating that hippocampus plays a major role in MCI classification. These findings are consistent with several previous studies [35] [36] [37] [38].

\subsection{Modularity Scores of FBNs}

For quantitatively evaluating the modularity of FBNs, we employ the signed modularity maximization algorithm to calculate the modularity scores of brain networks that constructed by different methods [39] [40]. In addition, to avoid the randomness of the results, we select 24 subjects equidistantly and calculate their FBN (with the optimal combination of parameters) modularity score. It can be observed from Figure 8 that the low-rank structure can improve the 


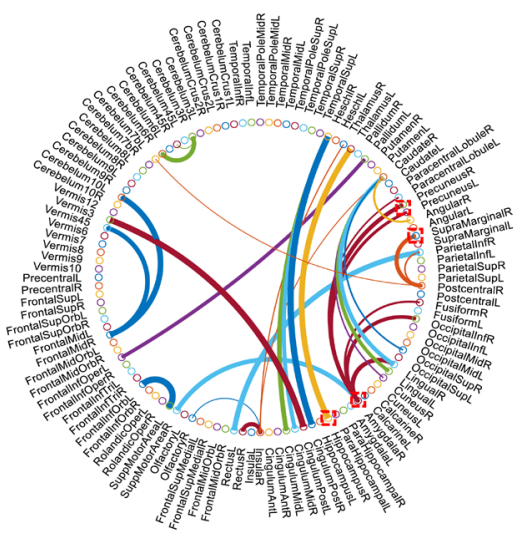

LR-PC

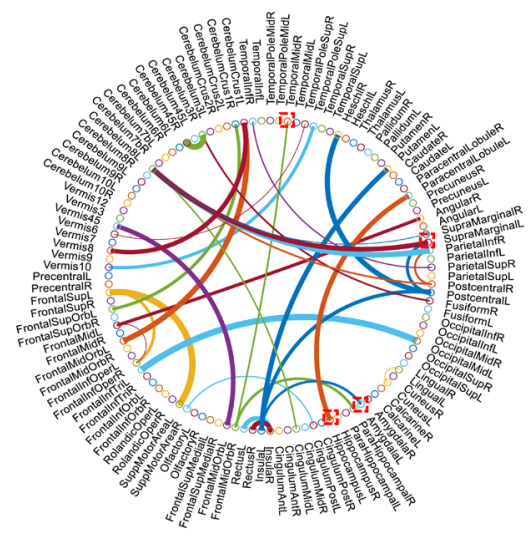

LR-SR

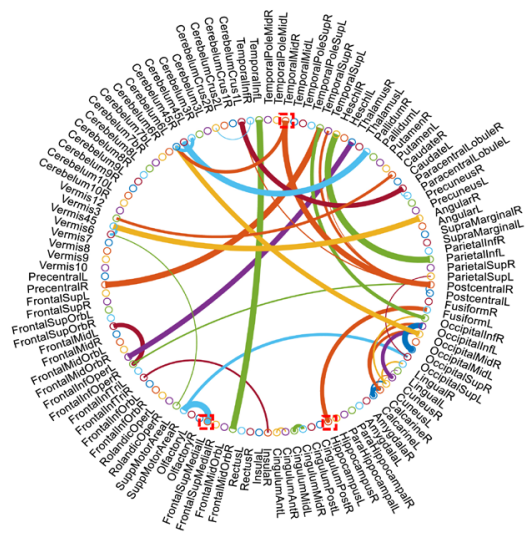

LR-HoFC

Figure 7. The most discriminative connections in FBNs that estimated by LR-PC, LR-SR and LR-HoFC, respectively. The nodes, indicated by red boxes, are related to the MCI identification according to previous study.

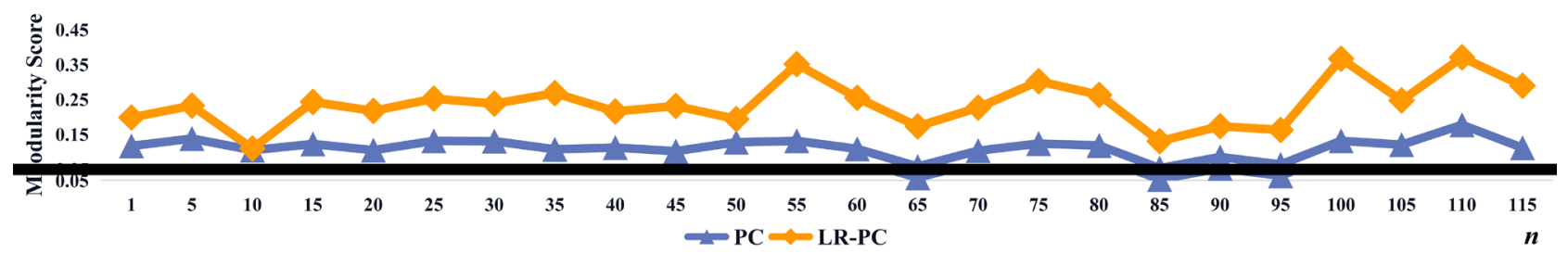

(a)

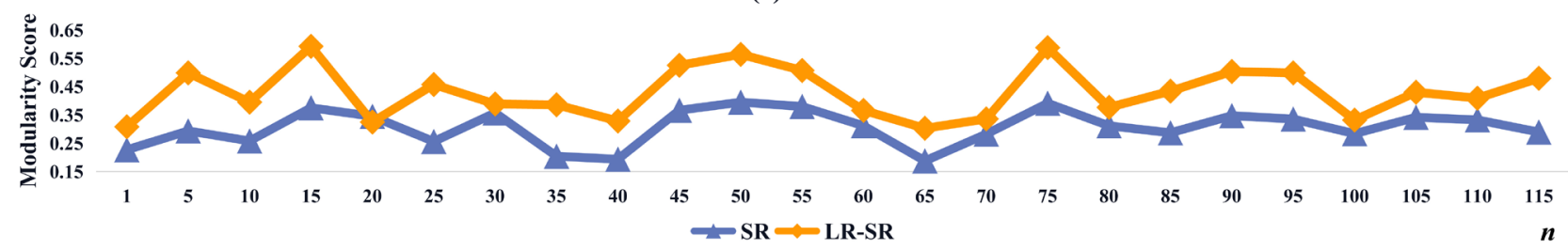

(b)

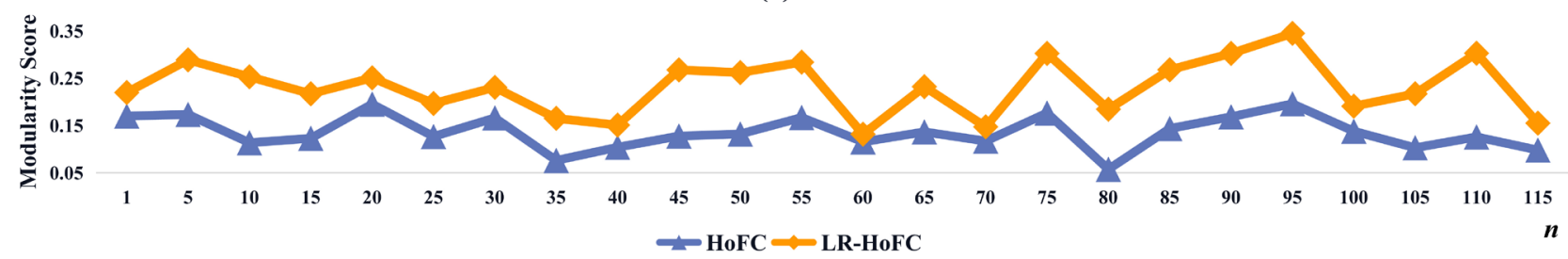

(c)

Figure 8. Modularity scores of networks constructed by different methods. And the abscissa indicates the label of the participant. (a) PC vs LR-PC; (b) SR vs LR-SR; (c) HoFC vs LR-HoFC.

modularity of FBN. Note that, the modularity of FBN that estimated by SR and LR-SR are generally higher than that by other methods, which indicates that moderate sparsity also can improve the modular structure of FBN.

\subsection{Comparison with State-of-the-Arts}

In Table 4, we briefly report the results of several state-of-the-art (SOTA) in the literature on the ADNI dataset compare with our method. As exhibited in Table 4 , the results achieved by our method are overall better than the four SOTA methods. Note that, even if the SEN and AUC respectively reported in [11] and [41] 
Table 4. Comparison with state-of-the-art methods for FBN-based MCI vs. NC classification with ADNI dataset. The top 2 best results are shown in bold.

\begin{tabular}{ccccccc}
\hline Method & Subject \# & ACC & SEN & SPE & F 1 & AUC \\
\hline Ritter et al. [42] & $151 \mathrm{MCI}+61 \mathrm{NC}$ & 0.7344 & - & - & 0.6945 & - \\
Kam et al. [43] & $49 \mathrm{MCI}+48 \mathrm{NC}$ & 0.7385 & 0.7391 & 0.7369 & - & - \\
Yang et al. [11] & $47 \mathrm{MCI}+29 \mathrm{NC}$ & $\mathbf{0 . 8 2 9 8}$ & 0.7662 & - & - & $\mathbf{0 . 9 4 0 6}$ \\
Chen et al. [41] & $45 \mathrm{MCI}+46 \mathrm{NC}$ & 0.8022 & $\mathbf{0 . 8 6 6 7}$ & 0.7391 & - & - \\
LR-PC (Ours) & $68 \mathrm{MCI}+69 \mathrm{NC}$ & 0.8219 & 0.8235 & $\mathbf{0 . 8 5 1 0}$ & $\mathbf{0 . 9 6 7 9}$ & 0.8794 \\
LR-SR (Ours) & $68 \mathrm{MCI}+69 \mathrm{NC}$ & $\mathbf{0 . 8 3 0 1}$ & $\mathbf{0 . 8 4 4 4}$ & $\mathbf{0 . 8 1 6 7}$ & $\mathbf{0 . 9 0 9 7}$ & $\mathbf{0 . 9 0 9 6}$ \\
LR-HoFC (Ours) & $68 \mathrm{MCI}+69 \mathrm{NC}$ & 0.7603 & 0.7976 & 0.7209 & 0.8000 & 0.8184 \\
\hline
\end{tabular}

are better than our method, their performances was on account of a relatively smaller dataset compared with our study.

\subsection{Limitations and Future Work}

There are some limitations in our current work. First, we only construct the low-rank FBNs based on the PC, SR and HoFC in this study. In fact, our proposed model can also combined with other FBN estimation methods [44], such as dynamic time warping distance (DTW) [45] and dynamic causal model (DCM) [46]. Second. The low-rank structure only enhance the modularity of FBN, but not make FBNs have an explicit modular structure. In the future, we plan to encode the modular structure with an elegant mathematical model for improving the quality of estimated FBNs, and further improve the classification accuracy.

\section{Conclusion}

In this paper, we propose a novel approach to estimate FBN by MF. More specifically, we implement this method with two steps. We firstly construct FBNs based on traditional methods, including PC, SR and HoFC. Then, we improve the modular structure of FBNs via MF model. For verifying the effectiveness of this method, we apply it to identify subjects with MCI/ASD from NCs. Compared with the baseline methods, our method has a better performance under five performance metrics. Moreover, we found that the FBNs, estimated by LR-PC, LR-SR and LR-HoFC, have higher modularity than the methods that do not take the low-rank structure of the FBNs into account.

\section{Supported}

This work was partly supported by National Natural Science Foundation of China (Nos. 61976110) and Natural Science Foundation of Shandong Province (Nos. ZR2018MF020).

\section{Conflicts of Interest}

The authors declare no conflicts of interest regarding the publication of this paper. 


\section{References}

[1] Castellanos, F., Martino, A.D., Craddock, R.C., Mehta, A.D. and Milham, M.P. (2013) Clinical Applications of the Functional Connectome. NeuroImage, 80, 527-540. https://doi.org/10.1016/j.neuroimage.2013.04.083

[2] Wee, C.Y., Yap, P.T. and Shen, D. (2014) Diagnosis of Autism Spectrum Disorders Using Temporally Distinct Resting-State Functional Connectivity Networks. $\mathrm{Hu}$ man Brain Mapping, 35, 3414-3430.

[3] Geschwind, D.H. and Levitt, P. (2007) Autism Spectrum Disorders: Developmental Disconnection Syndromes. Science Direct, 17, 103-111. https://doi.org/10.1016/j.conb.2007.01.009

[4] Chaudhuri, K.R. and Schapira, A.H. (2009) Non-Motor Symptoms of Parkinson's Disease: Dopaminergic Pathophysiology and Treatment. Lancet Neurology, 8, 464-474. https://doi.org/10.1016/S1474-4422(09)70068-7

[5] Supekar, K., Menon, V., Rubin, D., Musen, M. and Greicius, M. (2008) Network Analysis of Intrinsic Functional Brain Connectivity in Alzheimer's Disease. PLOS Computational Biology, 4, e1000100. https://doi.org/10.1371/journal.pcbi.1000100

[6] Goedert, M. and Spillantini, M.G. (2006) A Century of Alzheimer's Disease. Science, 314, 777-781. https://doi.org/10.1126/science.1132814

[7] Desikan, R.S., Cabral, H.J., Hess, C.P., Dillon, W.P., Glastonbury, C.M., Weiner, M.W., Schmansky, N.J., Greve, D.N., Salat, D.H., Buckner, R.L. and Fischl, B. (2009) Automated MRI Measures Identify Individuals with Mild Cognitive Impairment and Alzheimer's Disease. Brain, 132, 2048-2057.

https://doi.org/10.1093/brain/awp123

[8] Rombouts, S.A., Barkhof, F., Goekoop, R., Stam, C.J. and Scheltens, P. (2005) Altered Resting State Networks in Mild Cognitive Impairment and Mild Alzheimer's Disease: An fMRI Study. Human Brain Mapping, 26, 231-239.

https://doi.org/10.1002/hbm.20160

[9] Jie, B., Liu, M., Zhang, D. and Shen, D. (2018) Sub-network Kernels for Measuring Similarity of Brain Connectivity Networks in Disease Diagnosis. IEEE Transactions on Image Processing, 27, 2340-2353. https://doi.org/10.1109/TIP.2018.2799706

[10] Chen, X., Han, Z., Zhang, L., Shen, C., Lee, S. and Shen, D. (2017) Extraction of Dynamic Functional Connectivity from Brain Grey Matter and White Matter for mci Classification. Human Brain Mapping, 38, 5019-5034. https://doi.org/10.1002/hbm.23711

[11] Yang, P., Zhou, F., Ni, D., Xu, Y., Wang, T. and Lei, B. (2019) Fused Sparse Network Learning for Longitudinal Analysis of Mild Cognitive Impairment. IEEE Transactions on Cybernetics, 51, 1-14. https://doi.org/10.1109/TCYB.2019.2940526

[12] Bijsterbosch, J., Smith, S.M. and Beckmann, C.F. (2017) An Introduction to Resting State fMRI Functional Connectivity. Oxford University Press, Oxford.

[13] Macke, J.H., Opper, M. and Bethge, M. (2011) Common Input Explains Higher-Order Correlations and Entropy in a Simple Model of Neural Population Activity. Physical Review Letters, 106, Article ID: 208102. https://doi.org/10.1103/PhysRevLett.106.208102

[14] Plis, S.M., Sui, J., Lane, T., Roy, S., Clark, V.P., Potluru, V.K., Huster, R.J., Michael, A., Sponheim, S.R., Weisend, M.P. and Caljoun, V.D. (2014) High-Order Interactions Observed in Multi-Task Intrinsic Networks Are Dominant Indicators of Aberrant Brain Function in Schizophrenia. NeuroImage, 102, 35-48. https://doi.org/10.1016/j.neuroimage.2013.07.041 
[15] Chen, X., Zhang, H., Gao, Y. and Wee, Y.C. (2016) High-Order Resting-State Functional Connectivity Network for MCI Classification. Human Brain Mapping, 37, 3282-3296. https://doi.org/10.1002/hbm.23240

[16] Zhang, H., Chen, X., Shi, F., Li, G., Kim, M., Giannakopoulos, P., Haller, S. and Shen, D. (2016) Topographical Information-Based High-Order Functional Connectivity and Its Application in Abnormality Detection for Mild Cognitive Impairment. Journal of Alzheimer's Disease, 54, 1095-1112. https://doi.org/10.3233/JAD-160092

[17] Masaya, M., Daichi, K., Kenji, L. and Masayuki, M. (2018) Drawing Inspiration from Human Brain Networks: Construction of Interconnected Virtual Networks. Sensors, 18, 1133. https://doi.org/10.3390/s18041133

[18] Linda, G., Renken, R.J., Emi, S., Maurits, N.M. and Lorist, M.M. (2015) A Brain-Wide Study of Age-Related Changes in Functional Connectivity. Cerebral Cortex, 25, 1987-1999. https://doi.org/10.1093/cercor/bhu012

[19] Bertolero, M.A., Yeo, B.T.T. and D’Esposito, M. (2015) The Modular and Integrative Functional Architecture of the Human Brain. Proceedings of the National Academy of Sciences of the United States of America, 112, E6798-E6807.

https://doi.org/10.1073/pnas.1510619112

[20] Smith, S.M., Vidaurre, D., Beckmann, C.F., Glasser, M.F., Jenkinson, M., Miller, K.L., Nichols, T.E., Robinson, E.C., Salimi-Khorshidi, G., Woolrich, M.W., Barch, D.M., Ugurbil, K. and Van Essen, D.C. (2013) Functional Connectomics from Resting-State fMRI. Trends in Cognitive Sciences, 17, 666-682. https://doi.org/10.1016/j.tics.2013.09.016

[21] Lee, H., Lee, D.S., Kand, H., Kam, B. and Chung, M.K. (2012) Sparse Brain Network Recovery under Compressed Sensing. IEEE Transactions on Medical Imaging, 30, 1154-1165. https://doi.org/10.1109/TMI.2011.2140380

[22] Ehrlich andTobin, J. (2014) Networks of the Brain. Journal of Neuropathology EXperimental Neurology, 73, 1191. https://doi.org/10.1097/NEN.0000000000000142

[23] Zhou, Y., Zhang, L., Teng, S., Qiao, L. and Shen, D. (2018) "Improving Sparsity and Modularity of High-Order Functional Connectivity Networks for MCI and ASD Identification. Frontiers in Neuroscience, 12, 959.

https://doi.org/10.3389/fnins.2018.00959

[24] Yan, C. and Zang, Y. (2010) DPARSF: A MATLAB Toolbox for "Pipeline" Data Analysis of Resting-State fMRI. Frontiers in Systems Neuroscience, 4, 13. https://doi.org/10.3389/fnsys.2010.00013

[25] Tzourio-Mazoyer, N., Landeau, B., Papathanassiou, D., Crivello, F., Etard, O., Delcroix, N., Mazoyer, B. and Joliot, M. (2002) Automated Anatomical Labeling of Activations in SPM Using a Macroscopic Anatomical Parcellation of the MNI MRI Single-Subject Brain. NeuroImage, 15, 273-289.

https://doi.org/10.1006/nimg.2001.0978

[26] Qiao, L., Zhang, H., Kim, M., Teng, S., Zhang, L. and Shen, D. (2016) Estimating Functional Brain Networks by Incorporating a Modularity Prior. NeuroImage, 141, 399-407. https://doi.org/10.1016/j.neuroimage.2016.07.058

[27] Sapatinas, T. (2004) The Elements of Statistical Learning. Journal of the Royal Statistical Society: Series A (Statistics in Society), 167, 183-198. https://doi.org/10.1111/j.1467-985X.2004.298 11.x

[28] Kuo, F.Y. and Sloan, I.H. (2005) Lifting the Curse of Dimensionality. Notices of the American Mathematical Society, 52, 1320-1328.

[29] Robert and Tibshirani (1996) Regression Shrinkage and Selection via the Lasso. 
Journal of the Royal Statistical Society. Series B (Methodological), 58, 267-288. https://doi.org/10.1111/j.2517-6161.1996.tb02080.x

[30] Szenkovits, A., Meszlenyi, R., Buza, K., Gasko, N., Lung, R.I. and Suciu, M. (2017) Feature Selection with a Genetic Algorithm for Classification of Brain Imaging Data. Advances in Feature Selection for Data and Pattern Recognition, 138, 158-202. https://doi.org/10.1007/978-3-319-67588-6 10

[31] Chang, C.C. and Lin, C. (2011) Libsvm : a Library for Support Vector Machines. ACM Transactions on Intelligent Systems and Technology, 2, 1-27. https://doi.org/10.1145/1961189.1961199

[32] Wee, C.Y., Yap, P.T., Zhang, D., Wang, L. and Shen, D. (2013) Group-Constrained Sparse fMRI Connectivity Modeling for Mild Cognitive Impairment Identification. Brain Structure Function, 219, 641-656. https://doi.org/10.1007/s00429-013-0524-8

[33] Li, W., Wang, Z., Zhang, L., Qiao, L. and Shen, D. (2017) Remodeling Pearson's Correlation for Functional Brain Network Estimation and Autism Spectrum Disorder Identification. Frontiers in Neuroinformatics, 11. https://doi.org/10.3389/fninf.2017.00055

[34] Li, W., Qiao, L., Zhang, L., Wang, Z. and Shen, D. (2019) Functional Brain Network Estimation with Time Series Self-scrubbing. IEEE Journal of Biomedical and Health Informatics, 23, 2494-2504. https://doi.org/10.1109/JBHI.2019.2893880

[35] Albert, M.S., Dekosky, S.T., Dickson, D., Dubois, B., Feldman, H.H., Fox, N.C., Gamst, A., Holtzman, D.M., Jagust, W.J., Petersen, R.C., Snyder, P.J., Carrillo, M.C., Thies, B. and Phelps, C.H. (2011) The Diagnosis of Mild Cognitive Impairment Due to Alzheimer's Disease: Recommendations from the National Institute on Aging-Alzheimer's Association Workgroups on Diagnostic Guidelines for Alzheimer's Disease. Alzheimer's Dementia, 7, 270-279.

https://doi.org/10.1016/j.jalz.2011.03.008

[36] Kesler, S.R. (2014) Default Mode Network as a Potential Biomarker of Chemotherapy-Related Brain Injury. Neurobiology of Aging, 35, S11-S19. https://doi.org/10.1016/j.neurobiolaging.2014.03.036

[37] Zhu, X., IlvSuk, H., Lee, S.W. and Shen, D. (2016) Subspace Regularized Sparse Multitask Learning for Multiclass Neurodegenerative Disease Identification. IEEE Transactions on Bio Medical Engineering, 63, 607-618. https://doi.org/10.1109/TBME.2015.2466616

[38] He, Y., Wang, L., Zang, Y., Tian, L., Zhang, X., Li, K. and Jiang, T. (2007) Regional Coherence Changes in the Early Stages of Alzheimer's Disease: A Combined Structural and Resting-State Functional MRI Study. NeuroImage, 35, 488-500. https://doi.org/10.1016/j.neuroimage.2006.11.042

[39] Gomez, S., Jensen, P. and Arenas, A. (2009) Analysis of Community Structure in Networks of Correlated Data. Physical Review E, Statistical, Nonlinear, and Soft Matter Physics, 80, Article ID: 016114. https://doi.org/10.1103/PhysRevE.80.016114

[40] Rubinov, M. and Sporns, O. (2011) Weight-Conserving Characterization of Complex Functional Brain Networks. NeuroImage, 56, 2068-2079.

https://doi.org/10.1016/j.neuroimage.2011.03.069

[41] Chen, H., Zhang, Y., Zhang, L., Qiao, L. and Shen, D. (2021) Estimating Brain Functional Networks Based on Adaptively-Weighted fMRI Signals for MCI Identification. Frontiers in Aging Neuroscience, 12. https://doi.org/10.3389/fnagi.2020.595322

[42] Ritter, K., Schumacher, J., Weygandt, M., Buchert, R., Allefeld, C. and Haynes, J.D. (2015) Multimodal Prediction of Conversion to Alzheimer's Disease Based on In- 
complete Biomarkers. Alzheimer's Dementia: Diagnosis, Assessment Disease Monitoring, 1, 206-215. https://doi.org/10.1016/j.dadm.2015.01.006

[43] Kam, T.E., Zhang, H., Jiao, Z. and Shen, D. (2019) Deep Learning of Static and Dynamic Brain Functional Networks for Early MCI Detection. IEEE Transactions on Medical Imaging, 39, 478-487. https://doi.org/10.1109/TMI.2019.2928790

[44] Qiao, L., Zhang, L., Chen, S. and Shen, D. (2018) Data-Driven Graph Construction and Graph Learning: A Review. Neurocomputing, 312, 336-351. https://doi.org/10.1016/j.neucom.2018.05.084

[45] Meszlényi, R.J., Hermann, P., Buzr, K., Gal, V. and Vidnyanszky, Z. (2017) Resting State fMRI Functional Connectivity Analysis Using Dynamic Time Warping. Frontiers in Neuroscience, 11, 75-90. https://doi.org/10.3389/fnins.2017.00075

[46] Friston, K.J., Harrison, L. and Penny, W. (2003) Dynamic Causal Modelling. NeuroImage, 19, 1273-1302. https://doi.org/10.1016/S1053-8119(03)00202-7 